\section{Crescimento e estado nutricional de crianças e adolescentes de Presidente Prudente, São Paulo, Brasil}

\section{Growth and nutritional status of children and adolescents in the city of Presidente Prudente, State of São Paulo, Brazil}

Ismael Forte Freitas Júnior 1

Pedro Balikian Júnior 2

Letícia Kimie Miyashita 3

Cassiano Merussi Neiva 4

Sheila Cristhiane de Almeida Isidorio 5

\section{Abstract}

Objectives: to evaluate the weight, height, and Body Mass Index (BMI) of children and adolescents, in addition to the prevalence of overweight and obesity, in the city of Presidente Prudente, State of São Paulo, Brazil.

Methods: a sample of 1327 subjects $(692$ male and 635 female) aged 7-19 years were evaluated. The data were collected using the same equipment and following standard procedures described in literature. A Student's t test was used to compare independent variables $(p<0.05)$. Percentiles were calculated using SPSS, version 10.0 and plotted using Minitab, version 14.0. The 85 and 95 percentiles were used for classification of overweight and obesity, and were analysed in relation to two more internationally accepted studies.

Results: the weight and height are higher than in other studies carried out in Brazil and similar to those in a number of developed countries. The BMI figures revealed a high prevalence of overweight, and obesity, mainly in the male group between 7 and 10 years. This prevalence was less marked in the female group, especially among adolescents.

Conclusions: the growth of this sample is above that observed in other Brazilian regions, and comparable to some developed countries. However, the BMI indicates a high prevalence of overweight and obesity. Key words Growth, Nutritional status, Body mass index, Overweight, Obesity

\section{Resumo}

Objetivos: avaliar peso, altura, indice de massa corporal (IMC) e prevalência de sobrepeso e obesidade de crianças e adolescentes de Presidente Prudente, São Paulo, Brasil.

Métodos: foram avaliados 1327 jovens $(692$ masculino e 635 feminino) com idade de 7 a 19 anos. Os dados foram coletados com o mesmo equipamento e seguindo procedimento padrão descrito na literatura. $O$ teste $t$ de Student para variáveis independentes foi utilizado para as comparações entre sexo $(p<0,05), e$ foram calculados os percentis para cada idade. Utilizouse o programa SPSS, versão-10.0. A plotagem das curvas foi realizada no programa Minitab, versão-14.0. Os percentis 85 e 95 serviram para classificação de sobrepeso e de obesidade e foram analisados em relação aos dois estudos mais aceitos internacionalmente.

Resultados: o peso e a altura estão acima dos valores encontrados em outras regiões brasileiras e semelhantes a dos paises desenvolvidos. O IMC revelou alta prevalência de sobrepeso e obesidade, principalmente no sexo masculino, entre 7 e 10 anos. Esta prevalência foi menor no grupo feminino, especialmente entre as adolescentes.

Conclusões: a amostra apresenta padrão de crescimento acima de outras regiões brasileiras e comparáveis a países desenvolvidos, entretanto o IMC indica alta prevalência de sobrepeso e obesidade.

Palavras-chave Crescimento, Estado nutricional, Índice de massa corporal, Sobrepeso, Obesidade 


\section{Introdução}

Durante muitos anos boa parte das pesquisas em nutrição dedicaram-se a investigar aspectos relacionados à desnutrição e suas conseqüências para a saúde. ${ }^{1}$ Atualmente, o foco de atenção dos pesquisadores está voltado para o aumento desordenado do sobrepeso e da obesidade, que está afetando pessoas de todas as idades, sejam ricos, pobres, pertencentes a países desenvolvidos ou em desenvolvimento. ${ }^{2}$

No Brasil, dois estudos foram realizados com dados representativos nacionais. O primeiro foi o Estudo Nacional de Despesa Familiar (ENDEF), realizado nos anos de 1974/1975 e o segundo a Pesquisa Nacional sobre Saúde e Nutrição (PNSN), realizada em 1989.3

Mais recentemente foi realizado um levantamento representativo, em termos regionais porém, que foi a Pesquisa de Padrões de Vida, desenvolvida nas regiões Nordeste e Sudeste. 4

Esses estudos utilizaram o Índice de Massa Corporal (IMC) que, apesar do uso em crianças e adolescentes ser controverso, é o indicador mais utilizado no mundo todo para avaliar a prevalência de desordens nutricionais, especificamente a desnutrição, o sobrepeso e a obesidade. 5 A controvérsia existe principalmente porque o peso corporal e a altura variam com a idade e, inclusive, com o estado de maturação sexual. 6

Para classificação do IMC uma das referências mais utilizadas são os estudos publicados pelo National Center of Health Statistics (NCHS) dos Estados Unidos, e realizados pelo National Health and Nutritional Examination Survey (NHANES). ${ }^{7}$ A mais recente versão do NHANES, realizado entre os anos de 1999 e 2002, utilizou, respectivamente, os percentis 85 e 95 do IMC da população norte-americana como referência para sobrepeso e obesidade. ${ }^{8}$ Entretanto, pelo fato do citado estudo utilizar amostra da população norte-americana, seus resultados não são totalmente aceitos como referência internacional. ${ }^{9}$

A outra referência foi apresentada pelo Grupo de Trabalho em Obesidade Infantil (GTOI) e apresenta levantamentos feitos a partir de valores percentilares de dados internacionais e que são representativos da população de seis países: Brasil, Holanda, Inglaterra, China, Estados Unidos e Singapura. ${ }^{10}$ Esse estudo fornece dados de IMC para crianças e adolescentes de todas as idades que representam os valores $25 \mathrm{e}$ $30 \mathrm{~kg} / \mathrm{m}^{2}$ de IMC, considerados, respectivamente, limítrofes para classificação de sobrepeso e obesidade de indivíduos adultos.
Quanto aos estudos brasileiros, apesar da existência do ENDEF e do PNSN, a grande maioria dos trabalhos utiliza dados locais ou regionais. ${ }^{11}$

Particularmente em relação à região oeste do Estado de São Paulo, pode-se afirmar que, até o momento, não foi realizado qualquer estudo que possa ser utilizado como base para análise do crescimento e do estado nutricional da população local.

Este estudo tem como objetivo avaliar o peso, a altura e o índice de massa corporal de crianças e adolescentes de ambos os sexos, moradores na cidade de Presidente Prudente, e analisar as curvas indicativas de sobrepeso e de obesidade, com os valores utilizados internacionalmente como referência.

\section{Métodos}

Foram estudados 1327 indivíduos (692 do sexo masculino e 635 do sexo feminino), com idades de 7 a 19 anos. Os dados foram coletados durante os anos de 2000 a 2004, no Centro de Estudos e Laboratório de Avaliação e Prescrição de Atividades Motoras (CELAPAM) do Departamento de Educação Física da Faculdade de Ciências e Tecnologia (FCT) da Universidade Estadual Paulista (UNESP), Campus de Presidente Prudente, em São Paulo, Brasil, e referem-se a diversos estudos sobre crescimento, composição corporal e estado nutricional, realizados com crianças e adolescentes de escolas públicas e privadas, de programas de extensão desenvolvidos pela FCT/UNESP e de coletas de dados realizadas em programas comunitários locais.

Presidente Prudente é a maior e principal cidade desta região, denominada Alta Sorocabana, e apresenta grande concentração de moradores provenientes de diversas cidades próximas. Por esse motivo, acredita-se que uma amostra da população dessa cidade reflita o comportamento da população regional, o que contribuirá para se analisar o crescimento e o estado nutricional da população jovem dessa região

Todos os participantes ou seus responsáveis apresentaram consentimento para participar dos estudos.

Foram excluídos os indivíduos que apresentavam alguma disfunção ou alteração, motora ou postural, patológica ou não, que interferisse no resultado das medidas.

Para melhor confiabilidade dos dados, todos os avaliadores foram previamente treinados para as coletas e somente foram incluídos os valores obtidos com os mesmos equipamentos e seguindo os 
mesmos procedimentos metodológicos publicados na literatura. 12

O peso (P) foi obtido com balança de pêndulos, marca Filizzola, com precisão de $0,1 \mathrm{~kg}$. A altura (A) foi medida em estadiômetro com precisão de $0,1 \mathrm{~cm}$. Em ambos os casos os avaliados permaneciam em pé, peso distribuído igualmente sobre os pés, posição ortostática, calcanhares em contato um com o outro, braços descontraídos lateralmente ao tronco, cabeça posicionada no plano de Frankfurt e respirando normalmente.

O cálculo do Índice de Massa Corporal foi feito pela a divisão do valor do peso corporal, em kilogramas, pela altura, em metros, elevada ao quadrado $\left(\mathrm{IMC}=\mathrm{P} / \mathrm{A}^{2}\right)$

Foram utilizados dois procedimentos para análise dos dados. O primeiro foi o cálculo dos percentis $5,50,85$ e 95 das três variáveis. Para análise da prevalência de sobrepeso e de obesidade, decidiu-se pela utilização do IMC publicados pelo NHANES, versão I e II, e publicados por Frisancho, 13 e os dados do GTOI, publicados por Cole et al. 10

Os dados estudados são expressos na forma de médias, desvio padrão e intervalo de confiança de $95 \%$. O teste " $t$ " de Student para variáveis independentes foi utilizado para realizar as comparações entre os valores médios por sexo e idade. A significância estatística foi estabelecida em 5\%. Todas as análises foram realizadas utilizando o programa SPSS, versão 10.0 (SPSS Inc, Chicago, IL). As figuras foram feitas no pacote estatístico Minitab, versão 14 , com 0,7 graus para ajuste das curvas, utilizando, para isso, o recurso double experimental smotthing, do próprio software.

\section{Resultados}

Os valores médios das variáveis estudadas e as comparações entre sexo do grupo são apresentados na Tabela 1.

Verifica-se que a idade média do grupo não apresentou diferença estatística, entretanto os valores do peso, da altura e do IMC foram significantemente diferentes $(p<0,05)$ para o grupo do sexo masculino quando comparados ao sexo feminino.

Os valores médios e percentilares do peso corporal do sexo masculino e do feminino são registrados na Tabela 2. Verifica-se que, entre $10 \mathrm{e}$ 13 anos, o peso corporal médio dos rapazes passou de $36,8( \pm 8,5) \mathrm{kg}$ para $54,0( \pm 14,9) \mathrm{kg}$, diferença de $17,2 \mathrm{~kg}$, e entre 15 e 16 anos houve aumento $7,6 \mathrm{~kg}$ No caso do sexo feminino, os dados sugerem que o
Tabela 1

Média e desvio padrão de jovens de 7 a 19 anos

\begin{tabular}{lrr}
\hline & Masculino & \multicolumn{1}{c}{ Feminino } \\
\hline Idade (anos) & $14,1 \pm 3,6$ & $13,9 \pm 3,9$ \\
Peso $(\mathrm{kg})$ & $54,1 \pm 17,9 *$ & $47,7 \pm 15,4$ \\
Altura (cm) & $160,2 \pm 17,4$ * & $153,0 \pm 15,7$ \\
IMC (kg/m2) & $20,5 \pm 4,1$ * & $19,8 \pm 3,6$
\end{tabular}

* $p<0,5$ entre sexo; IMC = índice massa corporal.

maior aumento ocorre na faixa de 9 a 14 anos, passando de $30,6( \pm 7,15) \mathrm{kg}$ para $54,0( \pm 12,3) \mathrm{kg}$, com incremento de $23,4 \mathrm{~kg}$.

O comportamento do peso corporal de acordo com cada idade e as diferenças entre sexo podem ser melhor observados na Figura 1, principalmente entre 11 e 14 e após os 15 anos.

$\mathrm{Na}$ Tabela 3 são apresentados os valores da altura, para cada idade, dos indivíduos do sexo masculino e do sexo feminino. Observa-se que na faixa etária dos 10 aos 15 anos o incremento da altura dos rapazes foi de $32,5 \mathrm{~cm}$ e das moças de $26,6 \mathrm{~cm}$. O pico de crescimento ocorreu entre $12 \mathrm{e}$ 13 anos para ambos, com incremento de $8,8 \mathrm{~cm}$ no caso dos rapazes e de $11,8 \mathrm{~cm}$ no caso das moças.

O comportamento das curvas da altura pode ser melhor observado na Figura 2, e verifica-se que, no caso dos rapazes, o incremento chega até os 17 anos $(175,3 \pm 6,6 \mathrm{~cm})$. No caso das moças ocorre pequeno aumento entre os 14 anos $(162,0 \pm 7,0 \mathrm{~cm})$ e os 17 $\operatorname{anos}(165,4 \pm 6,8)$.

Quanto ao Índice de Massa Corporal $\left(\mathrm{kg} / \mathrm{m}^{2}\right)$, são apresentados na Tabela 4 os valores médios e percentilares para cada idade dos indivíduos do sexo masculino e do sexo feminino, respectivamente.

Observa-se aumentos variáveis com a idade em ambos os sexos, e no caso do sexo feminino, o IMC apresentou aumento constante dos 11 aos 16 anos (Tabela 4). Tal evidência pode ser melhor visualizada na Figura 3, inclusive, com valores maiores no grupo do sexo feminino entre 14 e 16 anos.

$\mathrm{Na}$ Tabela 5 são apresentados os resultados referentes à prevalência de sobrepeso e de obesidade de acordo com sexo e idade. 
Tabela 2

\begin{tabular}{|c|c|c|c|c|c|c|c|c|c|c|}
\hline \multirow[t]{3}{*}{ Idade (anos) } & \multicolumn{10}{|c|}{ Percentil } \\
\hline & \multicolumn{2}{|c|}{$\bar{X} \pm d p$} & \multicolumn{2}{|c|}{5} & \multicolumn{2}{|c|}{50} & \multicolumn{2}{|c|}{85} & \multicolumn{2}{|c|}{95} \\
\hline & $\mathrm{M}$ & $\mathrm{F}$ & $\mathrm{M}$ & $\mathrm{F}$ & $\mathrm{M}$ & $\mathrm{F}$ & M & $\mathrm{F}$ & $M$ & $\mathrm{~F}$ \\
\hline 7 & (32) $33,6 \pm 9,3$ & (38) $30,2 \pm 7,8$ & 20,8 & 20,7 & 31,8 & 28,1 & 42,6 & 39,7 & 52,5 & 48,1 \\
\hline 8 & (28) $32,1 \pm 8,5$ & (36) $32,1 \pm 8,9$ & 21,5 & 21,4 & 31,2 & 29,1 & 40,0 & 44,7 & 52,2 & 49,4 \\
\hline 9 & (41) $33,8 \pm 11,4$ & (40) $30,6 \pm 7,1$ & 23,1 & 21,6 & 30,2 & 28,9 & 46,3 & 39,8 & 59,9 & 43,3 \\
\hline 10 & (40) $36,8 \pm 8,5$ & (33) $36,4 \pm 12,6$ & 24,7 & 23,0 & 34,8 & 33,2 & 47,7 & 53,6 & 53,5 & 63,3 \\
\hline 11 & (46) $41,8 \pm 11,2$ & (55) $35,5 \pm 10,8$ & 30,6 & 22,1 & 39,1 & 32,4 & 47,7 & 47,3 & 70,4 & 59,1 \\
\hline 12 & (46) $45,7 \pm 10,8$ & (50) $39,5 \pm 12,8$ & 28,4 & 21,2 & 46,5 & 39,6 & 56,7 & 54,3 & 64,1 & 61,4 \\
\hline 13 & (60) $54,0 \pm 14,9$ & (41) $48,8 \pm 11,5$ & 33,3 & 29,2 & 52,5 & 48,1 & 61,8 & 58,0 & 85,5 & 76,9 \\
\hline 14 & (61) $57,1 \pm 19,3$ & (44) $54,0 \pm 12,3$ & 31,8 & 39,8 & 54,2 & 51,3 & 83,2 & 68,7 & 92,4 & 81,3 \\
\hline 15 & (53) $57,3 \pm 15,4$ & (41) $55,5 \pm 10,0$ & 34,3 & 42,2 & 54,7 & 55,2 & 66,1 & 68,5 & 95,2 & 72,6 \\
\hline 16 & (53) $64,9 \pm 10,6$ & (26) $57,7 \pm 11,8$ & 48,4 & 39,9 & 63,6 & 56,0 & 76,2 & 68,1 & 84,5 & 88,1 \\
\hline 17 & (69) $67,3 \pm 10,9$ & (72) $60,0 \pm 10,8$ & 52,5 & 46,4 & 65,6 & 57,0 & 76,0 & 68,2 & 86,8 & 85,2 \\
\hline 18 & (84) $67,7 \pm 13,1$ & (77) $57,3 \pm 9,4$ & 51,5 & 44,0 & 65,7 & 57,0 & 78,1 & 65,2 & 95,9 & 77,2 \\
\hline 19 & (79) $63,8 \pm 13,9$ & (82) $58,7 \pm 9,6$ & 44,0 & 46,0 & 62,4 & 58,0 & 79,3 & 69,6 & 82,8 & 80,6 \\
\hline
\end{tabular}

M=Masculino; F=Feminino; (n).

Figura 1

Peso corporal (média e IC95\%) de rapazes (linha contínua) e de moças (linha tracejada) com idades entre 7 e 19 anos.

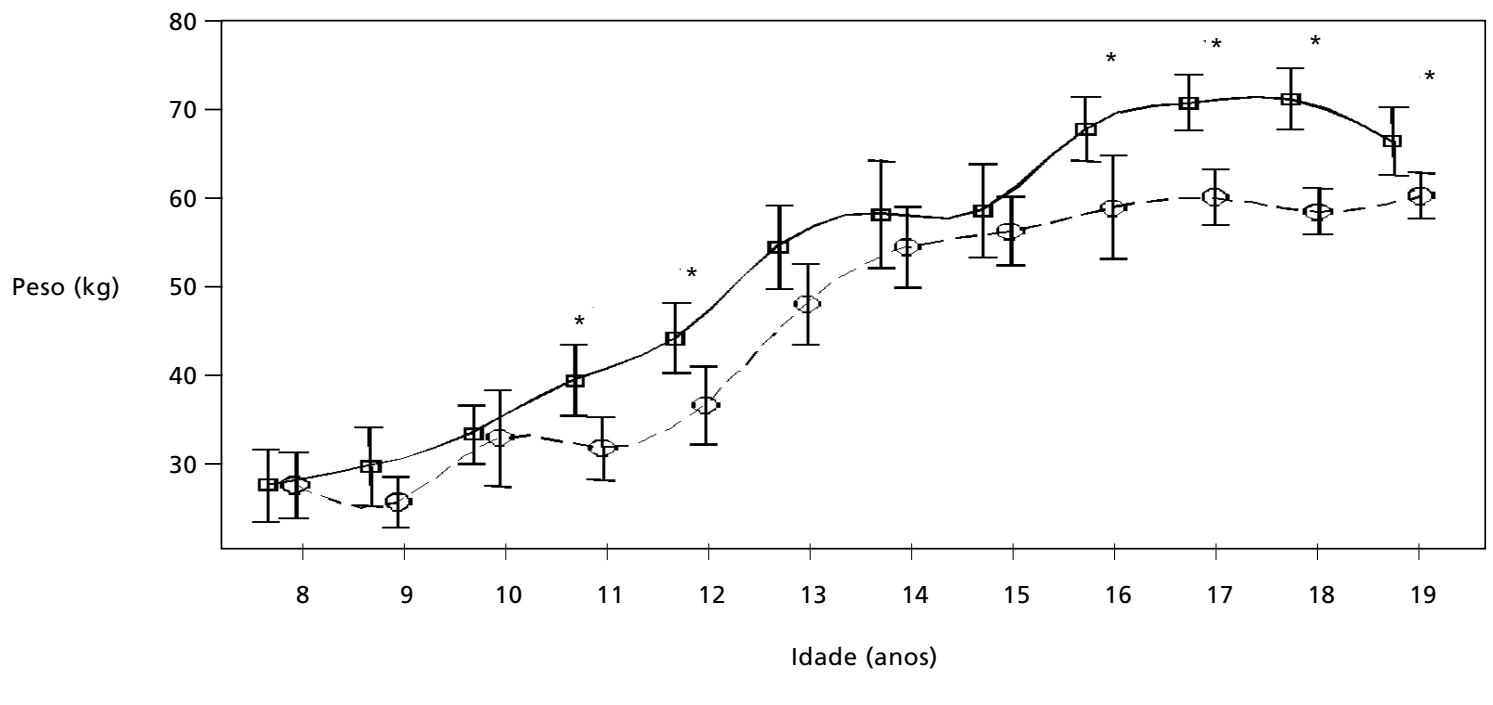

$* p<0,05$ 
Valores percentilares da altura $(\mathrm{cm})$ de indivíduos de 7 a 19 anos do sexo masculino e do sexo feminino.

\begin{tabular}{|c|c|c|c|c|c|c|c|c|c|c|}
\hline \multirow[t]{3}{*}{ Idade (anos) } & \multicolumn{10}{|c|}{ Percentil } \\
\hline & \multicolumn{2}{|c|}{$\bar{X} \pm d p$} & \multicolumn{2}{|c|}{5} & \multicolumn{2}{|c|}{50} & \multicolumn{2}{|c|}{85} & \multicolumn{2}{|c|}{95} \\
\hline & M & $\mathrm{F}$ & M & $\mathrm{F}$ & M & $\mathrm{F}$ & M & $\mathrm{F}$ & M & $\mathrm{F}$ \\
\hline 7 & (32) $133,0 \pm 12,5$ & (38) $130,8 \pm 10,6$ & 117,5 & 118,6 & 128,4 & 127,3 & 148,7 & 141,5 & 161,7 & 153,5 \\
\hline 8 & (28) $132,7 \pm 9,1$ & (36) $134,7 \pm 12,0$ & 120,1 & 118,1 & 132,8 & 134,0 & 140,1 & 152,0 & 156,8 & 158,5 \\
\hline 9 & (41) $136,0 \pm 7,6$ & (40) $134,2 \pm 7,0$ & 126,2 & 122,1 & 134,6 & 133,9 & 144,7 & 142,1 & 152,3 & 144,3 \\
\hline 10 & (40) $140,7 \pm 6,4$ & (33) $138,8 \pm 9,4$ & 130,6 & 119,2 & 140,7 & 138,9 & 147,0 & 149,3 & 150,0 & 157,0 \\
\hline 11 & (46) $146,8 \pm 6,0$ & (55) $140,1 \pm 9,7$ & 138,1 & 122,1 & 146,6 & 142,2 & 153,8 & 150,0 & 155,3 & 154,7 \\
\hline 12 & (46) $153,3 \pm 10,0$ & (50) $45,5 \pm 15,6$ & 134,0 & 119,6 & 153,8 & 149,5 & 165,0 & 162,3 & 168,6 & 168,4 \\
\hline 13 & (60) $162,1 \pm 10,1$ & (41) $157,3 \pm 8,4$ & 148,0 & 142,3 & 161,9 & 160,0 & 173,9 & 164,4 & 182,0 & 167,9 \\
\hline 14 & (61) $164,9 \pm 15,0$ & (44) $162,0 \pm 7,0$ & 134,4 & 151,1 & 164,0 & 162,4 & 181,0 & 170,0 & 189,8 & 172,5 \\
\hline 15 & (53) $167,7 \pm 10,8$ & (41) $160,0 \pm 4,9$ & 143,8 & 150,2 & 171,0 & 160,7 & 176,7 & 165,5 & 179,9 & 167,9 \\
\hline 16 & (53) $173,2 \pm 7,9$ & (26) $161,9 \pm 6,4$ & 157,9 & 149,2 & 174,0 & 163,0 & 181,0 & 169,0 & 185,5 & 172,1 \\
\hline 17 & (69) $175,3 \pm 6,6$ & (72) $165,4 \pm 6,8$ & 163,6 & 156,1 & 176,0 & 164,6 & 182,5 & 171,1 & 185,5 & 180,1 \\
\hline 18 & (84) $173,6 \pm 8,6$ & (77) $163,4 \pm 7,1$ & 158,3 & 152,5 & 174,0 & 162,0 & 182,3 & 170,0 & 186,4 & 176,2 \\
\hline 19 & (79) $169,4 \pm 9,9$ & (82) $165,4 \pm 9,3$ & 152,0 & 153,2 & 171,0 & 163,3 & 179,0 & 175,6 & 184,0 & 185,7 \\
\hline
\end{tabular}

$M=$ Masculino; F=Feminino; (n).

Figura 2

Comportamento da altura (média e IC95\%) de rapazes (linha contínua) e moças (linha tracejada) com idades entre 7 e 19 anos, representado pelo valor médio e pelo Intervalo de Confiança de $95 \%$.

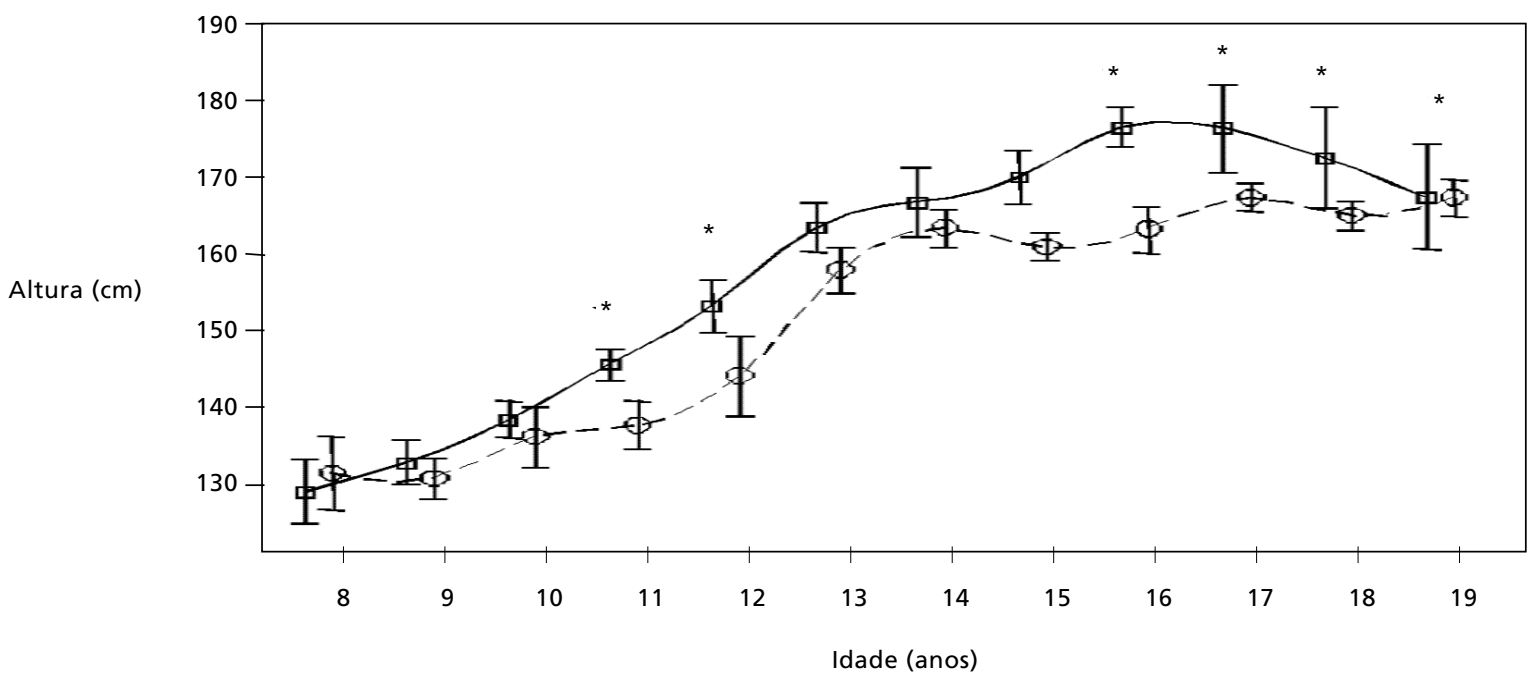

$* p<0,05$ 
Tabela 4

\begin{tabular}{|c|c|c|c|c|c|c|c|c|c|c|c|}
\hline \multirow[t]{3}{*}{ Idade (anos) } & \multicolumn{11}{|c|}{ Percentil } \\
\hline & \multicolumn{3}{|c|}{$\bar{X} \pm d p$} & \multicolumn{2}{|c|}{5} & \multicolumn{2}{|c|}{50} & \multicolumn{2}{|c|}{85} & \multicolumn{2}{|c|}{95} \\
\hline & $\mathrm{M}$ & & $\mathrm{F}$ & M & $\mathrm{F}$ & M & $\mathrm{F}$ & M & $\mathrm{F}$ & $\mathrm{M}$ & $\mathrm{F}$ \\
\hline 7 & (32) $18,8 \pm 3,5$ & (38) & $17,4 \pm 2,9$ & 14,9 & 14,1 & 17,3 & 16,8 & 23,1 & 19,9 & 25,3 & 24,1 \\
\hline 8 & (28) $18,0 \pm 3,1$ & (36) & $17,4 \pm 2,6$ & 13,8 & 14,0 & 17,1 & 17,2 & 20,8 & 19,3 & 25,4 & 23,5 \\
\hline 9 & (41) $18,0 \pm 4,3$ & $(40)$ & $16,9 \pm 3,3$ & 14,0 & 11,0 & 16,9 & 16,9 & 22,2 & 20,8 & 26,2 & 22,1 \\
\hline 10 & (40) $18,5 \pm 3,7$ & (33) & $18,5 \pm 4,1$ & 14,4 & 14,3 & 16,5 & 17,0 & 22,9 & 23,1 & 26,1 & 27,9 \\
\hline 11 & (46) $19,3 \pm 4,3$ & (55) & $17,8 \pm 3,5$ & 13,9 & 14,3 & 18,2 & 16,7 & 21,8 & 21,8 & 31,0 & 26,4 \\
\hline 12 & (46) $19,2 \pm 3,2$ & (50) & $18,1 \pm 3,0$ & 13,8 & 13,4 & 18,7 & 17,3 & 23,2 & 21,4 & 24,5 & 24,1 \\
\hline 13 & (60) $20,4 \pm 4,4$ & $(41)$ & $19,5 \pm 3,4$ & 14,8 & 14,6 & 19,0 & 19,0 & 24,5 & 23,6 & 28,5 & 27,1 \\
\hline 14 & (61) $20,5 \pm 4,6$ & (44) & $20,4 \pm 3,8$ & 15,1 & 15,9 & 19,6 & 19,8 & 25,0 & 24,8 & 31,7 & 28,9 \\
\hline 15 & (53) $20,2 \pm 4.6$ & (41) & $21,6 \pm 3,4$ & 16,0 & 17,1 & 18,9 & 21,5 & 22,4 & 25,4 & 34,6 & 29,0 \\
\hline 16 & (53) $21,6 \pm 2,6$ & (26) & $21,9 \pm 3,7$ & 18,0 & 16,5 & 21,3 & 20,7 & 24,0 & 25,4 & 26,8 & 31,5 \\
\hline 17 & (69) $21,8 \pm 2,3$ & (72) & $21,8 \pm 3,1$ & 18,1 & 17,7 & 21,3 & 21,6 & 25,0 & 24,7 & 27,9 & 27,3 \\
\hline 18 & (84) $22,4 \pm 3,5$ & (77) & $21,4 \pm 3,0$ & 17,5 & 17,6 & 21,9 & 21,1 & 25,3 & 23,9 & 30,0 & 28,3 \\
\hline 19 & (79) $22,1 \pm 3,9$ & $(82)$ & $21,4 \pm 2,0$ & 17,8 & 18,3 & 21,7 & 21,4 & 24,5 & 23,3 & 27,5 & 25,3 \\
\hline
\end{tabular}

$M=$ Masculino; $F=$ Feminino; $(n)$.

Figura 3

Comportamento do Índice de Massa Corporal (IMC), média e IC95\%, de rapazes (linha contínua) e de moças (linha tracejada) com idades entre 7 e 19 anos.\%.

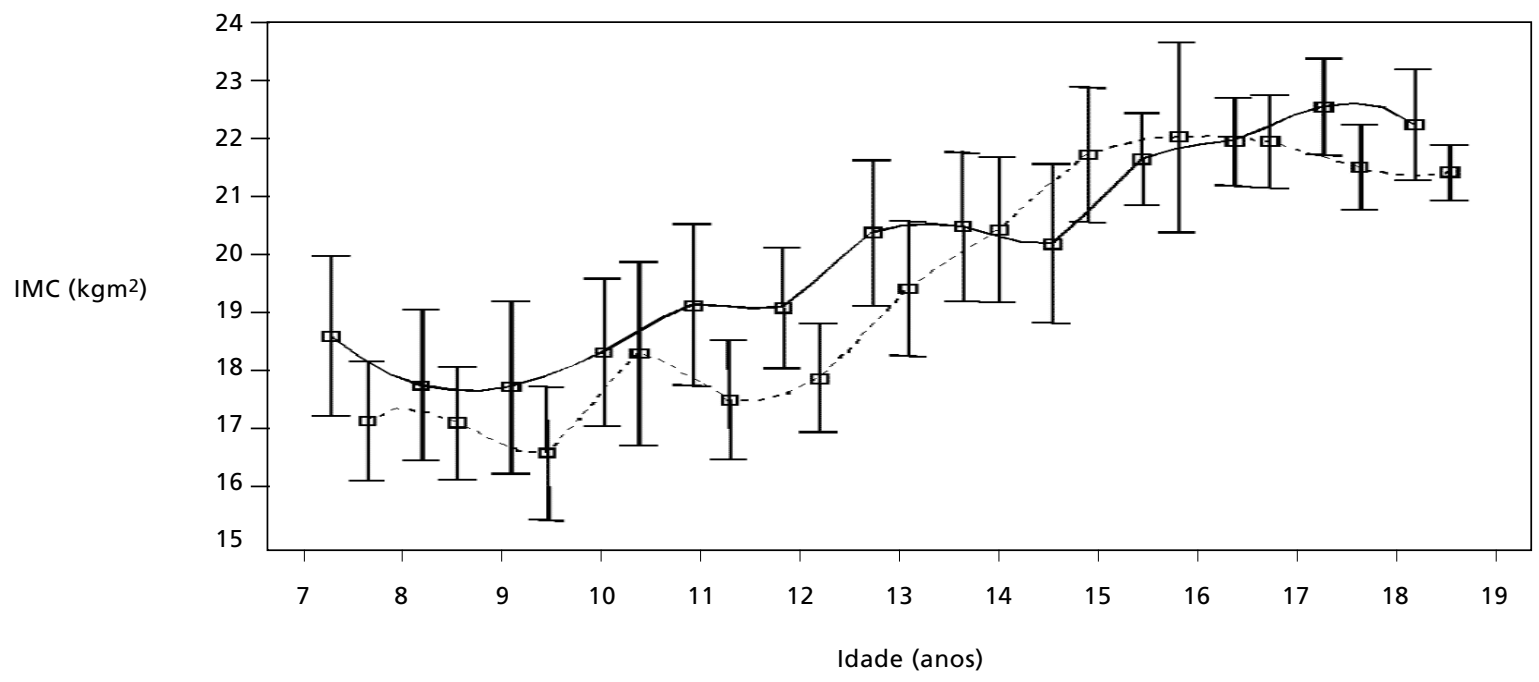


Tabela 5

\begin{tabular}{|c|c|c|c|c|c|c|c|c|}
\hline \multirow[t]{2}{*}{ Idade (anos) } & \multicolumn{2}{|c|}{$\mathrm{N}$} & \multicolumn{2}{|c|}{ Sobrepeso } & \multicolumn{2}{|c|}{ Obesidade } & \multicolumn{2}{|c|}{ Sobrepeso+Obesidade } \\
\hline & $\mathrm{M}$ & $\mathrm{F}$ & $M$ & $\mathrm{~F}$ & $M$ & $\mathrm{~F}$ & $M$ & $\mathrm{~F}$ \\
\hline 7 & 32 & 38 & 9,4 & 23,7 & 37,5 & 13,2 & 46,9 & 36,8 \\
\hline 8 & 28 & 36 & 14,3 & 30,6 & 25,0 & 11,1 & 39,3 & 41,7 \\
\hline 9 & 41 & 40 & 7,3 & 17,5 & 14,6 & 2,5 & 22,0 & 20,0 \\
\hline 10 & 40 & 33 & 25,0 & 18,2 & 12,5 & 9,1 & 37,5 & 27,3 \\
\hline 11 & 46 & 55 & 13,0 & 9,1 & 10,9 & 7,3 & 23,9 & 16,4 \\
\hline 12 & 46 & 50 & 19,6 & 14,0 & 2,2 & 0,0 & 21,7 & 14,0 \\
\hline 13 & 60 & 41 & 20,0 & 17,1 & 8,3 & 2,4 & 28,3 & 19,5 \\
\hline 14 & 61 & 44 & 8,2 & 13,6 & 11,5 & 4,5 & 19,7 & 18,2 \\
\hline 15 & 53 & 41 & 5,7 & 14,6 & 5,7 & 4,9 & 11,3 & 19,5 \\
\hline 16 & 53 & 26 & 13,2 & 19,2 & 1,9 & 3,8 & 15,1 & 23,1 \\
\hline 17 & 69 & 72 & 15,9 & 11,1 & 1,4 & 2,8 & 17,4 & 13,9 \\
\hline 18 & 84 & 77 & 13,1 & 7,8 & 3,6 & 1,3 & 16,7 & 9,1 \\
\hline
\end{tabular}

$M=$ Masculino; $F=$ Feminino.

\section{Discussão}

O peso, a altura e o IMC são medidas simples, confiáveis e amplamente utilizadas por profissionais da saúde pública e área clínica, por servirem como indicadores das condições de saúde da população, pela sua relação com a gordura corporal não só de adultos, mas de crianças e adolescentes, 14 e porque servem como instrumento para análise das condições sanitárias e de variações socioeconômicas de uma dada população. 15

Quando se trata de estudos envolvendo grandes amostras, como é o caso de estudos epidemiológicos, é recomendado utilizar medidas simples, que seriam aplicadas facilmente, sem a necessidade de equipamentos que possam causar diferenças nos resultados, levando ao aumento na margem de erro, causada por fatores como experiência do avaliador ou marca e modelo do equipamento utilizado. 16

Outro importante aspecto a ser considerado é que esse estudo fez uso de dados de IMC de dois levantamentos amplamente utilizados internacionalmente, para analisar o estado nutricional (sobrepeso e obesidade) dos participantes.

A análise do peso e da altura de jovens do sexo masculino comparados com os do sexo feminino sugere que, ambos apresentam pico de aumento entre 11 e 15 anos, com os rapazes mantendo valores maiores, curva mais homogênea e constante até, aproximadamente, os 17 anos. Comportamentos semelhantes do peso e, principalmente, da altura nessa faixa etária, também foram revelados nas curvas publicadas pelo $\mathrm{NCHS},{ }^{7}$ referente à população norte-americana.

Ao se analisar os dados do peso e da altura em relação às curvas do NCHS, para crianças de sete a nove anos de ambos os sexos, verificou-se que os avaliados no presente estudo apresentaram valores médios situando-se entre os percentis 75 e 95 dos norte-americanos (NCHS). Nas idades subseqüentes os meninos apresentaram padrão de crescimento ligeiramente acima com pequena variação aos 19 anos.

O comportamento do peso e da altura dos meninos com idades entre 7 e 10 anos deste grupo estudado também apresentou diferenças em relação a outro trabalho publicado recentemente, envolvendo amostra de jovens neo-zelandezes. ${ }^{17}$

No caso das meninas, o incremento do peso e da altura acompanhou, com pequenas variações, o padrão de aumento dos outros dois estudos citados, que envolveram jovens de mesma idade.7,17

O comportamento do peso, da altura e do IMC também foi analisado em relação a dados internacionais de jovens de 13 e 15 anos, de ambos os sexos, referentes a treze países europeus, além de Israel e Estados Unidos. Na idade de 13 anos, verificou-se que os valores médios do peso $(54,0 \mathrm{~kg}) \mathrm{e}$ 
do IMC $\left(20,4 \mathrm{~kg} / \mathrm{m}^{2}\right)$ dos rapazes do presente estudo situaram-se acima daqueles apresentados por jovens europeus e norte-americanos. A altura, por sua vez, apresentou valores médios similares em relação aos europeus. Sete dos treze países investigados apresentaram valores de peso e de IMC abaixo dos jovens desse estudo. 18

Quanto às moças, verificou-se que o peso aos 13 anos apresenta valores maiores somente que o das jovens de dois países (Israel e Lituania) e, aos 15 anos, os resultados foram similares, sendo maior que o das jovens de sete dos treze países europeus. A altura, por sua vez, apresentou, para ambas as idades, resultados médios menores que o das jovens de todos os países. Como reflexo desse maior peso e menor altura, o IMC das moças de 13 anos do presente estudo, foi maior que o das jovens de 11 países e, aos 15 anos, somente não foram maiores que os valores médios das jovens norte-americanas. 19

A análise dos resultados do peso e da altura da amostra do presente trabalho em relação à amostra de jovens brasileiros, revela que o peso e altura foram maiores em todas as idades, tanto para rapazes como para moças, do que para jovens escolares do município do Rio de Janeiro, 20 e para jovens da cidade de Londrina, Paraná, em estudo publicado em 1993.21

Tanto para o sexo masculino como para o feminino, os casos de maior aumento de peso ocorreram a partir dos 12 anos nos percentis 5 e 50 . Tais variações podem ser explicadas pela preocupação dos adolescentes com o controle do excesso de peso, principalmente daqueles que se encontram nos limites superiores (percentis 85 e 95) e, no sentido oposto, o maior incremento não linear do peso após os 12 anos, daqueles que se encontram no limite inferior (percentil 5), pode estar refletindo a melhoria do estado nutricional.

Esta evidência pode indicar que a população jovem dessa região do estado de São Paulo apresenta padrão de crescimento similar a alguns países desenvolvidos, principalmente no caso dos mais jovens e do sexo masculino, e maior peso e altura que os jovens de outras localidades brasileiras, o que não significa que apresentam melhores condições nutricionais, pois para se chegar a essa conclusão, somente a medida do peso e da altura não são suficientes.

Mesmo não existindo forma de classificação de sobrepeso e de obesidade aceita internacionalmente para ser aplicada a crianças e adolescentes, tanto o Center for Disease Control and Prevention como o European Childhood Obesity Group recomendam o uso do IMC como indicador de estado nutricional para uso clínico, bem como em pesquisas epidemiológicas. 18

Tomando-se como referência os estudos do GTOI ${ }^{10}$ e do NCHS, ${ }^{7}$ verifica-se que a amostra do presente estudo apresenta grande proporção de rapazes com sobrepeso e obesidade $(25,0 \%)$, ao passo que entre as moças a prevalência foi menor $(21,6 \%)$. Essa menor prevalência entre as moças em relação aos rapazes também foi observada em estudo publicado recentemente, com jovens da cidade do Rio de Janeiro, ${ }^{20}$ e pode ser reflexo da preocupação desse grupo em relação a aparência estética. ${ }^{22}$ Outra possível explicação seriam as variações na maturação sexual, que interferem na composição corporal. 23 Tal evidência foi observada em amostras de jovens chineses, cuja prevalência do sobrepeso e da obesidade diminui no período da puberdade, principalmene em moças. 24

A maior prevalência de sobrepeso e obesidade no grupo investigado pelo presente estudo foi observada entre 7 e 10 anos; para meninos, foi de $36,4 \%$ e, para meninas, foi de $31,4 \%$. Esses valores estão próximos aos $37,1 \%$ de sobrepeso em amostras de crianças brasileiras de 6 a 11 anos da cidade de Niterói, Rio de Janeiro, 25 bem como da prevalência observada em alguns países Europeus que foi, no máximo, de 36\%, verificada em meninos italianos de nove anos. 5

No caso dos adolescentes do sexo masculino, a prevalência de sobrepeso e de obesidade nessa faixa etária também foi bastante alta tanto entre 11 e 14 anos $(23,4 \%)$, como acima dessa idade $(15,1 \%)$. No caso das moças, a prevalência foi de $17,0 \%$ entre 11 e 14 anos e de 16,4\% acima dessa faixa etária. Essa proporção está acima dos $8,5 \%$ observada na cidade de Niterói, Rio de Janeiro 25 e dos 6,9\% dos escolares da própria cidade do Rio de Janeiro. ${ }^{26}$

A análise da prevalência de sobrepeso em relação a amostras de jovens de mesma idade avaliados por toda Europa revelou que tanto os rapazes quanto as moças do presente estudo apresentam valores maiores de IMC do que os dos jovens de diversos países europeus, que foi de 10 a $20 \%$ em amostras de países do Norte europeu e de 20 a $35 \%$ no Sul desse Continente. 5

Os resultados desse estudo indicam que a amostra da população jovem dessa Região do estado de São Paulo apresenta valores médios de peso e de altura maior do que indivíduos de mesma idade e sexo, investigados em outras regiões brasileiras, 20,21,25,26 e valores próximos a jovens de mesma idade de países desenvolvidos, como Estados Unidos, ${ }^{7}$ Nova Zelândia17 e vários países europeus. 19 
Outra evidência observada chama atenção: a semelhança do peso e da altura, não significa que a amostra local apresenta a mesma proporção de peso para altura. Os achados em relação ao IMC revelam que o excesso de peso parece estar atingindo proporções alarmantes nessa população, principalmente entre os mais jovens. Tal evidência merece atenção especial por diversas razões, incluindo o fato que o alto IMC na infância e na adolescência pode estar indicando sobrepeso ou obesidade na idade adulta, ${ }^{27}$ além de aumento da susceptibilidade para o desenvolvimento de morbidades futuras 28 e, como conseqüências negativas mais imediatas, estão aquelas relacionadas a aspectos psicológicos e comportamentais. 5 Tal fato sugere que medidas preventivas devam ser adotadas no sentido de

\section{Referências}

1. Wang Y, Monteiro C, Popkin BM. Trends of obesity and underweight in older children and adolescents in the United States, Brazil, China, and Russia. Am J Clin Nutr. 2002; 75: 971-7.

2. Sawaya AL, Roberts S. Stunting and future risk of obesity: principal physiological mechanisms. Cad Saúde Pública. 2003; 19 (Suppl 1): S21-S8.

3. Monteiro CA, Benicio MH, Iunes R, Gouveia NdaC, Taddei JA, Cardoso MA. ENDEF and PNSN: trends in physical growth of Brazilian children. Cad Saúde Pública. 1993; 9 (Suppl 1): 85-95.

4. Monteiro CA, D'A Benicio MH, Conde WL, Popkin BM Shifting obesity trends in Brazil. Eur J Clin Nutr. 2000;54: 342-6.

5. Lobstein T, Baur L, Uauy R. Obesity in children and young people: a crisis in public health. Obes Rev .2004;5 (Suppl 1): 4-104.

6. Guo SS, Chumlea WC, Roche AF, Siervogel RM. Age- and maturity-related changes in body composition during adolescence into adulthood: the fels longitudinal study. Int J Obes Relat Metab Disord. 1997; 21: 1167-75.

7. NCHS (National Health and Nutrition Examination Survey). National Health and Nutrition Examination Survey Monitoring the Nation's Health [on line] [2005 Feb 10]. Available from: URL http://www.cdc.gov/nchs/ nhanes.htm.

8. Hedley AA, Ogden CL, Johnson CL, Carroll MD, Curtin LR, Flegal KM. Prevalence of overweight and obesity among US children, adolescents, and adults, 1999-2002. JAMA. 2004; 291: 2847-50.

9. Flegal KM, Ogden CL, Wei R, Kuczmarski RL, Johnson CL. Prevalence of overweight in US children: comparison of US growth charts from the Centers for Disease Control and Prevention with other reference values for body mass index. Am J Clin Nutr. 2001; 73: 1086-93. promover melhorias qualitativas em termos alimentares e incentivo a prática de atividades físicas, principalmente em locais públicos e nas escolas, que são os dois ambientes mais freqüentados por crianças e adolescentes.

Mesmo considerando que o presente trabalho é um estudo transversal e não longitudinal e, por isso, não reflete o estado de crescimento de um mesmo indivíduo ao longo do tempo, tais dados poderão servir como base para futuras análises que possam ser feitas em nível regiões ou como referência para comparação de amostras de outras regiões brasileiras, podendo, dentro de seu limite de aplicabilidade, ser útil não só em termos acadêmicos, mas clínico, dada a significativa amostra envolvida.

10. Cole TJ, Bellizzi MC, Flegal KM, Dietz WH. Establishing a standard definition for child overweight and obesity worldwide: international survey. Br Med J. 2000; 320: 1240-3.

11. Guedes DP. Composição corporal: princípios, técnicas e aplicações. Londrina: APEF; 1994.

12. Lohman TG, Roche AF, Martorell R. Anthropometric standardization reference manual. Champaign, IL: Human Kinetics Books; 1988.

13. Frisancho A. Anthropometric standards for the assessment of growth and nutritional status. Michigan: The University of Michigan; 1999.

14. Garza C, De Onis M. A new international growth reference for young children. Am J Clin Nutr. 1999; 70: 169S-72S.

15. Guo SS, Chumlea WC. Tracking of Body Mass Index in children in relation to overweight in adulthood. Am J Clin Nutr. 1999; 70: 145S-8S.

16. Malina RM, Katzmarzyk PT. Validity of the Body Mass Index as an indicator of the risk and presence of overweight in adolescents. Am J Clin Nutr. 1999; 70: 131S-6S.

17. Duncan E, Schofield G, Duncan S, Kolt G, Rush E. Ethnicity and body fatness in New Zealanders. N Z Med. J 2004; 117: U913.

18. Taylor RW, Jones IE, Williams SM, Goulding A. Body fat percentages measured by dual-energy X-ray absorptiometry corresponding to recently recommended Body Mass Index cutoffs for overweight and obesity in children and adolescents aged 3-18 y. Am J Clin Nutr. 2002;76: 1416-21.

19. Lissau I, Overpeck MD, Ruan WJ, Due P, Holstein BE, Hediger ML. Body Mass Index and overweight in adolescents in 13 European countries, Israel, and the United States. Arch Pediatr Adolesc Med. 2004; 158: 27-33.

20. Anjos LA, Castro IR, Engstrom EM, Azevedo AM. Growth and nutritional status in a probabilistic sample of schoolchildren from Rio de Janeiro, 1999. Cad Saúde Pública. 2003; 19 (Suppl 1): S171-S9. 
21. Guedes DP, Guedes JE. Growth and motor performance of schoolchildren from the city of Londrina, Parana, Brazil. Cad Saúde Pública. 1993; 9 (Suppl 1): 58-70.

22. Pietrobelli A, Faith MS, Allison DB, Gallagher D, Chiumello G, Heymsfield SB. Body Mass Index as a measure of adiposity among children and adolescents: a validation study. J Pediatr. 1998; 132: 204-10.

23. Monteiro CA, Conde WL, Popkin BM. A tendência secula da obesidade segundo estratos sociais: Nordeste e Sudeste do Brasil, 1975-1989-1997. Arq Bras Endocrinol e Metab. 1999; 43: 186-94.

24. Wang Y, Ge K, Popkin BM. Tracking of Body Mass Index from childhood to adolescence: a 6 -y follow-up study in China. Am J Clin Nutr. 2000; 72: 1018-24.

25. Marins VM, Almeida RM. Undernutrition prevalence and social determinants in children aged 0-59 months, Niteroi, Brazil. Ann Hum Biol. 2002; 29: 609-18.
26. da Veiga GV, Dias PC, dos Anjos LA. A comparison of distribution curves of Body Mass Index from Brazil and the United States for assessing overweight and obesity in Brazilian adolescents. Rev Panam Salud Publica. 2001; 10: 79-85.

27. Wright CM, Parker L, Lamont D, Craft AW. Implications of childhood obesity for adult health: findings from thousand families cohort study. BMJ. 2001; 323: 1280-4.

28. Reich A, Muller G, Gelbrich G, Deutscher K, Godicke R, Kiess W. Obesity and blood pressure-results from the examination of 2365 schoolchildren in Germany. Int J Obes Relat Metab Disord. 2003; 27: 1459-64.

Recebido em 16 de março de 2006

Versão final apresentada em 3 de fevereiro de 2008

Aprovado em 3 de março de 2008 